\title{
Recognition of Acoustic Emission Signal based on the Algorithms of TDNN and GMM
}

\author{
Aidong Deng ${ }^{1}$, Hao Cao ${ }^{2, *}$, Hang Tong ${ }^{3}, \mathrm{Li} \mathrm{Zhao}^{4}, \mathrm{Kang} \mathrm{Qin}^{1}, \mathrm{Xi} \mathrm{Yan}^{1}$ \\ ${ }^{1}$ National Engineering Research Center of Turbo-generator Vibration, Southeast University, Nanjing 210096, China \\ ${ }^{2}$ Hunan Electric Power Corporation Research Institute, Changsha 410007, China \\ ${ }^{3}$ Huadian Electric Power Science Research Institute, Hangzhou 310030, China \\ ${ }^{4}$ School of Information Science and Engineering, Southeast University, Nanjing 210096, China
}

Received: 26 Apr. 2013, Revised: 27 Aug. 2013, Accepted: 29 Aug. 2013

Published online: 1 Mar. 2014

\begin{abstract}
Friction fault diagnosis of rotating machinery based on acoustic emission (AE) technique is a research hotspot in recent years. The rotating machinery will produce multi-source noise during the operation process, so how to correctly identify the friction acoustic emission signals has become a key factor for accurate diagnosis of the fault. In this paper, it proposes a Gaussian mixed model (GMM) based on an embedded time delay neural network (TDNN) to identify friction acoustic emission signals. It comprehensively utilizes the advantages of the learning ability of time delay neural network about data structure and data distribution presentation capability of Gaussian mixture model. Time delay neural network fully exploits the time-ordered of eigenvector set, makes the maximum likelihood probability more reasonable which needs to assume that the variables are independent of each other through the transformation of the time delay network and uses them for the training as a whole with the criteria of maximum likelihood (ML) probability. During the training process, the parameters of Gaussian mixture model and neural network update alternately. The average amplitude, maximum amplitude, amplitude dynamic range, the Hurst exponent and approximate entropy (ApEn) of friction acoustic emission signals are selected as the characteristic parameters of fault recognition and these five parameters constitutes the input parameters vector of the identification model. Through the verification the AE signals of different friction states collected on the rotor test bed, the experimental results show that the identification method of rotor friction acoustic emission signals of Gaussian mixed model based on embedded time delay neural network is an effective mean of identification with high recognition efficiency.
\end{abstract}

Keywords: Acoustic Emission; Signal Recognition; Time Delay Neural Network ;Gaussian Mixture Model

\section{Introduction}

Acoustic emission(AE) technique with its high sensitivity provides a new method for the diagnosis of rotating machinery friction fault. However, due to the prominent noise problem faced by the $\mathrm{AE}$, especially the relatively poor working environment of rotating machinery and the multi-source noise produced by the device during the operation process coupled with the attenuation and frequency dispersion distortion during the structure-borne process of AE signals, the identification of useful friction AE signals becomes very difficult $[1,2,3,4]$. Therefore, the research of more effective AE identification methods has important practical significance to the promotion of AE friction fault diagnosis.
Among the pattern recognition methods, the method based on Gaussian mixture background model (GMM) has achieved an increasing attention of people $[5,6,7]$ with advantages such as high recognition rate, simple training, low training data quantity requirement and it has become a mainstream recognition method. Support vector machines (SVM) and the factor analysis method [8,9] based on GMM super-vector represents the latest achievements of GMM method. GMM can approach to any distribution model owing to its good capability of data distribution representation ability as long as there are enough items and enough training data. But, GMM can only approach to the data distribution, it can do nothing to the changes of underlying data distribution and can not make any transformation to the data i.e. it can not change the data distribution; moreover, because we usually take

\footnotetext{
*Corresponding author e-mail: caohao82@gmail.com
} 
each variance matrix of Gaussian distribution as the diagonal matrix when we select a GMM model, this assumption is not that reasonable for it requires sufficient Gaussian mixture items. Neural network also occupies an important position in pattern recognition such as multi-layer perceptron (MLP), radial basis network and time delay neural network, and all of them have achieved very good identification effect $[10,11]$. Neural network transforms the eigenvector and makes the eigenvector got from transformation approach to the target vector in some way through the study especially in the case that TDNN is widely used in signal processing and pattern recognition $[12,13,14]$ which takes full advantage of timing information of eigenvector sequence.

It puts forward a kind of friction acoustic emission signal recognition method combining GMM and TDNN after giving full consideration to the respective advantages of GMM and TDNN in this paper. This method embeds TDNN to GMM, takes the maximum likelihood probability as the common principle of training TDNN and GMM so that TDNN can learn the time information of eigenvector, maps the eigenvector sets to the subspace which can increase the likelihood probability of target friction acoustic emission signal and reduces the impact of the unreasonable assumption of eigenvector independence. We propose a two-stage learning method to update the parameters of GMM and TDNN alternately. Experimental results show that the recognition rate of our proposed method exceeds the Gaussian mixture background model, which verifies validity of our proposed identification method.

\section{Use GMM with TDNN to identify the friction acoustic emission signals}

GMM can be regarded as a kind of Hidden Markov Model (HMM) with status number of 1 . An M-order GMM probability density function is the weighted sum of M Gaussian probability density functions which can be expressed by the following form $[5,6]$ :

$$
p\left(x_{t} \mid \lambda\right)=\sum_{i=1}^{M} p_{i} b_{i}\left(x_{t}\right)
$$

The $x_{t}$ here is a D-dimensional random vector which is usually able to characterize every eigenvector of characteristic parameters of all kinds of AE signals in the recognition of friction acoustic emission signals; $b_{i}\left(x_{t}\right), i=1,2, \ldots, M$ stands for member density function; $p_{t}, i=1,2, \ldots, M$ stands for mixed weights. Every member density function is a Gaussian function with D-dimensional variable, the mean vector $u_{t}$ and the covariance matrix $\sum_{i}$, and its form is as follows:

$$
b_{i}\left(x_{t}\right)=\frac{\exp \left\{-\frac{1}{2}\left(x_{t}-u_{i}\right)^{T} \sum_{i}^{-1}\left(x_{t}-u_{i}\right)\right\}}{(2 \pi)^{D / 2}\left|\sum_{i}\right|^{1 / 2}}
$$

Thereinto the mixed weights satisfy the condition that: $\sum_{i=1}^{M} p_{i}=1$.

The complete Gaussian mixture model is composed of all the member density function mean vectors, covariance matrix and mixed weight parameters. These parameters are gathered together and expressed as follows:

$$
\sum_{i=1}^{M} p_{i}=1, i=1,2, \ldots, M
$$

Generally speaking, the training and identification data of AE signal is relatively less, therefore, we usually assume that each Gaussian mixture density covariance matrix is diagonal during the actual use of GMM, and we train the parameters through the Expectation Maximization (EM) criteria which is as follows:

Firstly, select the initial values of $p_{i}, u_{i}, \sum_{i}$ in formula (3), calculate the class posterior probability:

$$
p\left(i \mid x_{t}, \lambda\right)=\frac{p_{i} b_{i}\left(x_{t}\right)}{\sum_{k=1}^{M} p_{k} b_{k}\left(x_{t}\right)}
$$

Then, calculate the weight coefficient, mean value and variance estimate value according to the following formula. Weight coefficient:

$$
\overline{p_{i}}=\frac{1}{N} \sum_{t=1}^{N} p\left(i \mid x_{t}, \lambda\right)
$$

Mean value:

$$
\overline{u_{i}}=\frac{\sum_{t=1}^{N} p\left(i \mid x_{t}, \lambda\right) x_{t}}{\sum_{t=1}^{N} p\left(i \mid x_{t}, \lambda\right)}
$$

Variance

$$
\bar{\sum}_{i}^{2}=\frac{\sum_{t=1}^{N} p\left(i \mid x_{t}, \lambda\right) x_{t}^{2}}{\sum_{t=1}^{N} p\left(i \mid x_{t}, \lambda\right)}-\bar{u}_{i}^{2}
$$

Substituting $\overline{p_{i}}, \overline{u_{i}}, \bar{\Sigma}_{i}$ to formula (1) and (2), we can get a new parameter value in formula (3), and repeat this operation, we can work out the model parameter $\lambda_{i}$.

When it goes to the identification of rotor friction acoustic emission signal, each type of rotor friction acoustic emission signal is expressed by a GMM and its model parameter $\lambda_{i}$.

For sequences with $\mathrm{T}$ testing vectors $X=X_{1}, X_{2}, \ldots, X_{T}$, its GMM probability can be written as:

$$
P(X \mid \lambda)=\prod_{t=1}^{T} p\left(X_{t} \mid \lambda\right)
$$

In logarithmic domain, it can be expressed as:

$$
L(X \mid \lambda)=\log P(X \mid \lambda)=\sum_{t=1}^{T} \log p\left(X_{t} \mid \lambda\right)
$$


Use Bayes' theorem in the identification process, among the $\mathrm{N}$ unknown rotor friction acoustic emission signal models, the maximum likelihood model's corresponding rotor friction acoustic emission signal is the very identification result:

$$
i^{*}=\underset{1 \leq i \leq N}{\arg \max } L\left(X \mid \lambda_{i}\right)
$$

Time delay network has been widely used in pattern recognition, TDNN network has the ability to associate and compare the current input and the previous inputs. Thus, the network inputs must be in chronological order, both TDNN networks with feedback and without feedback are acceptable, and in this paper, TDNN network without feedback is mainly used as is shown in Figure 1. The time delayed eigenvector, as the input of neural network, shall be nonlinearly transformed by it and then linearly weighted to form the output which will be compared with the eigenvector, and the commonly used criteria is MMSE. TDNN is a multilayer perceptron network, so the method of training multi perceptron networks can be also applied to training TDNN network.

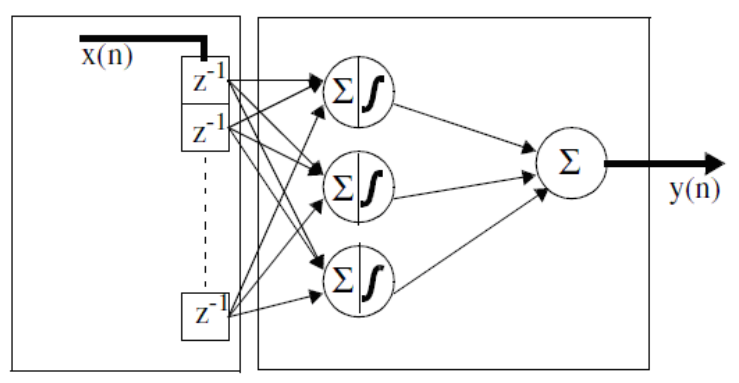

Fig. 1: Time delay neural network model without feedback

TDNN has the ability of excavating the time information of eigenvector sequences, so on the basis of synthesizing the respective advantages of GMM and the TDNN network, we propose a scheme with the TDNN network embedded in GMM. It comprehensively utilizes TDNN's learning ability about data structure and GMM's capability of data distribution presentation capability. They are trained as a whole that they jointly use the maximum likelihood probability criteria so that the neural network can learn the time information of eigenvector sequence, thus the learning results will proceed toward the direction of increasing the target likelihood probability. Next we will instruct the proposed identification method in several aspects such as training and recognizing models, training methods and TDNN convergence etc.

During the training, after the time delayed eigenvector enters the TDNN network, the TDNN network learns the structure of the eigenvector set and extracts the time information of the eigenvector sequence. And then provides the study results to GMM in the form of residual eigenvectors (i.e. the difference between the input vector and the output of TDNN network), trains GMM model with EM criteria and makes use of Back Propagation algorithm (BP) to update the weight coefficient of TDNN network. The criteria of TDNN network and the studying and training of GMM model are both maximum likelihood probability. In this way, through learning, the residual distribution is likely proceeding towards the direction of enhancing the likelihood probability.

During the recognition, the eigenvector enters the TDNN network after the delay. Because the TDNN network has studied the structure and timing information of the feature space, it will makes appropriate transformation of the eigenvector after its entrance, and then provides it to GMM model. Owing to the study, TDNN network transformation will play a role of enhancing the target model likelihood probability and reducing that of the non-target model.

We shall train this general purpose background model and the rotor friction acoustic emission signal model by a two-stage method, and the training process of TDNN network and that of GMM model will be proceeded alternately. We usually use EM method to train the general purpose background model GMM. TDNN is a multi-layer Perceptron, so we adopt the BP method with momentum to train the TDNN. The training process is described as follows:

1. Determine the GMM model and TDNN network structure;

2. Given the convergence condition and maximum iterating times;

3. Randomly choose the initial parameters of the TDNN network and GMM models;

4. Fix TDNN network parameters, input the eigenvector to TDNN network, get all of the residual vectors;

5. Amend the weight coefficients, mean vectors and variances of each Gaussian distribution of GMM model by EM method [5] ;

6. Utilize the amendatory weight coefficients, mean vectors and variances of each Gaussian distribution of GMM model, input the residual to get a likelihood probability, amend TDNN network parameters by the BP method with momentum;

7. Determine whether it meets the training stop condition, if so, stop the training, if not, turn to step 4 . We adopt a two-step iteration method to get the model parameters, so the GMM weight coefficient, mean vector and covariance matrix need to be fixed when we iterate the neural network parameters. Neural network iteration generally seeks a minimum value, and during the computations, addition is more convenient than multiplication, so we take the negative logarithm of neural network parameters and we can get that:

$$
\omega_{i j}^{*}=\underset{\omega_{i j}}{\arg \min }\left(-\sum_{t=1}^{N} \ln p\left(\left(x_{t}-o_{t}\right) \mid \lambda\right)\right)
$$


The $p\left(\left(x_{t}-o_{t}\right) \mid \lambda\right)$ here please refer to formula (1), $o_{t}$ stands for Neural network output and $\mathrm{N}$ stands for the number of training samples. The BP method with momentum [15] is able to accelerate the iterative convergence process and deal with local minimum problems more efficiently.

$$
\Delta \omega_{i j}^{k}(m+1)=\gamma \Delta \omega_{i j}^{k}(m)-\left.(1-\gamma) \alpha \frac{\partial F(x)}{\partial \omega_{i j}^{k}}\right|_{\omega_{i j}^{k}=\omega_{i j}^{k}(m)}
$$

Thereinto, $\Delta \omega_{i j}^{k}(m+1)=\omega_{i j}^{k}(m+1)-\omega_{i j}^{k}(m)$,

stands for the number of iterations which can be selected according to the actual situation; $\mathrm{k}$ stands for the serial number of the neural network layer, $\gamma$ stands for the inertia coefficient and here $\alpha$ stands for the iteration step. $F(x)=-\ln p((x-o) \mid \lambda)$, the subscript $\mathrm{t}$ is omitted for convenience.

Next we will calculate $: \frac{\partial F(x)}{\partial \omega_{i j}^{k}}$.

$$
\begin{gathered}
\frac{\partial F(x)}{\partial \omega_{i j}^{k}}=\frac{\partial F(x)}{\partial y_{i}^{k}} \frac{\partial y_{i}^{k}}{\partial \omega_{i j}^{k}} \\
\frac{\partial y_{i}^{k}}{\partial \omega_{i j}^{k}}=o_{j}^{k-1}
\end{gathered}
$$

Calculate the output layer $\frac{\partial F(x)}{\partial y_{i}^{k}}$ :

$$
\begin{aligned}
& \frac{\partial F(x)}{\partial y_{i}^{k}}=-\frac{1}{p((x-o) \mid \lambda)} \frac{\partial p((x-o) \mid \lambda)}{\partial o_{i}^{k}} \frac{\partial o_{i}^{k}}{y_{i}^{k}} \\
& =-\frac{f^{\prime}\left(y_{i}^{k}\right)}{p((x-o) \mid \lambda)} \partial\left(\sum_{n=1}^{M} p_{n} c_{n} e^{-\frac{1}{2}\left(x-o-u_{n}\right)^{T} \sum_{n}^{-1}\left(x-o-u_{n}\right)}\right) / \partial o_{i}^{k} \\
& =-\frac{f^{\prime}\left(y_{i}^{k}\right)}{p((x-o) \mid \lambda)} \sum_{n=1}^{M} p_{n} c_{n}\left(\frac{a_{n}\left(x-o-u_{n}\right)}{\sigma_{n, i}^{2}}\left(x_{i}-o_{i}-u_{n, i}\right)\right)
\end{aligned}
$$

\section{Thereinto:}

$a_{n}\left(x-o-u_{n}\right)=e^{-\frac{1}{2}\left(x-o-u_{n}\right)^{T} \sum_{n}^{-1}\left(x-o-u_{n}\right)}$

$c_{n}=\frac{1}{(2 \pi)^{D / 2}\left|\Sigma_{n}\right|^{1 / 2}}$

Calculate for the hidden layer $\frac{\partial F(x)}{\partial y_{i}^{k}}$ :

$$
\begin{aligned}
& \frac{\partial F(x)}{\partial y_{i}^{k}}=\sum_{j} \frac{\partial F(x)}{\partial y_{j}^{k+1}} \frac{\partial y_{j}^{k+1}}{\partial y_{i}^{k}}=\sum_{j} \frac{\partial F(x)}{\partial y_{j}^{k+1}} \frac{\partial\left(\sum_{n} \omega_{j n}^{k+1} o_{n}^{k}\right)}{\partial y_{i}^{k}} \\
& =\sum_{j} \frac{\partial F(x)}{\partial y_{j}^{k+1}} \frac{\partial o_{i}^{k}}{\partial y_{i}^{k}} \omega_{j i}^{k+1}=f^{\prime}\left(y_{i}^{k}\right) \sum_{j} \frac{\partial F(x)}{\partial y_{i}^{k+1}} \omega_{j i}^{k+1}
\end{aligned}
$$

Due to the backward inversion, before the calculation process for $\frac{\partial F(x)}{\partial y_{i}^{k}}, \frac{\partial F(x)}{\partial y_{i}^{k+1}}$ has been known, and substitute $\frac{\partial F(x)}{\partial y_{i}^{k+1}}$ into formula (16) then we can get $\frac{\partial F(x)}{\partial y_{i}^{k}}$.

At last, we shall simply prove that this training method can obtain a local maximum point of the model. Because during the EM training process, each obtained likelihood probability is monotone non-descending, if we select the step size factor as well in the training of TDNN network to make the likelihood probability after each iteration monotonous non-decreasing, the monotone non-decreasing sequence must have limit. Therefore, this can explain that the training process can converge to a local maximum point.

Of course, in order to avoid the premature convergence to local maximum point, at the beginning of step selection, we can choose a bigger one, and at this time, there are certain advantages to the oscillation of the training process instead. But the followed iterative step size must be well controlled, or the training process may be too long or even diverging.

The results got from the above method are the local maximum points in theory either it is GMM or TDNN network. Therefore, we need to start the training with many initial values and step mechanism in order to achieve better model parameters.

\section{Rotor friction acoustic emission signal characteristic extraction}

After the design of the recognition model, acoustic emission characteristic parameters which can characterize different friction conditions must also be determined. Next, we will explain the selection basis of the characteristic parameters through theory and experiments.

This experiment adopts 3-bearing 2-cross-rotor system, 3 bearings are plain bearings which can effectively simulate rotor imbalance, axis misalignment, rub-impact and other faults as is shown in Figure 2. The friction of static and dynamic components of the rotor system can be simulated through rubbing bracket fixed on the base of the rotor bed, as is shown in Figure 3. Rubbing stent is installed between bearing 1 and 2, and we can simulate different levels of rubbing faults by adjusting the rub screw which is located in the flank of the disc and faces the shaft center along the shaft radial. The AE signals generated by the rubbing source transmit to both sides of the AE sensor through the wave guide plate. Set the sampling frequency of $1 \mathrm{MHz}$ and rotational speed of $1480 \mathrm{r} / \mathrm{min}$ in the experiment. AE waveforms and spectra of several friction states are shown as Figure 4.

\subsection{Time domain characteristic parameters}

It can be seen from Figure 4 that the waveform is the noise signal and its amplitude is very small in no friction state. After the occurrence of the friction, the signal amplitude changes significantly and increases apparently with the increasing magnitude of the friction. In the frequency domain, noise energy is concentrated near $3 \mathrm{kHz}$, the friction AE signal frequency is above $5 \mathrm{kHz}$, and the more serious the friction is, the richer the high frequency component appears. We can intuitively distinguish the friction state by the energy amplitude of $\mathrm{AE}$ signal, and according to this characteristic, we choose 


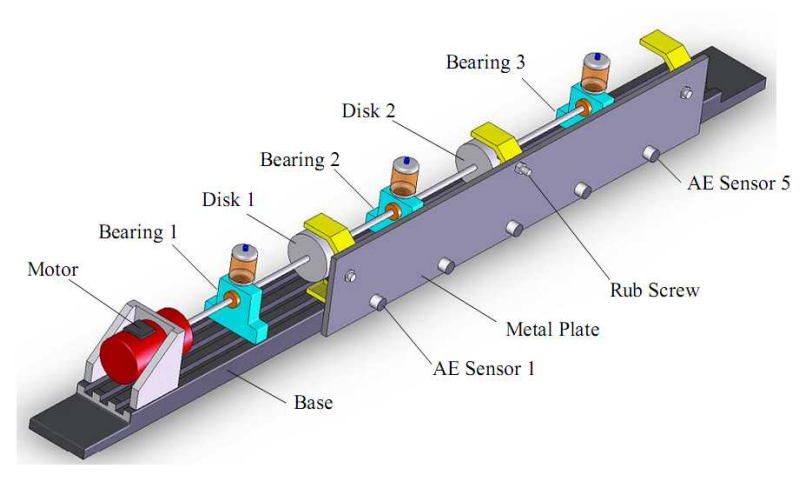

Fig. 2: Rotor rubbing test bed

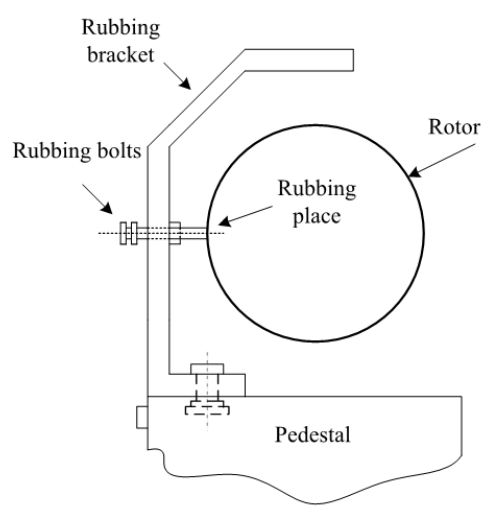

Fig. 3: Rubbing bracket

the average amplitude, maximum amplitude, amplitude dynamic range these three time domain parameters as the identification characteristic parameters of AE signal.

\subsection{Hurst index}

Owing to its reliable characterization of the extent of long memory, the Hurst index has been applied in machinery, equipment condition monitoring and fault diagnosis field. Studies have shown that the Hurst index can reflect the characteristics of acoustic emission signals under different frictional strength and it can be used as the basis of distinguishing the occurrence of the friction and its intensity changing trend [16] .

For the original time sequence $\left\{x_{t}\right\}_{t=1}^{\mathrm{T}}$, select the scale length $n$, divide the entire time series into $M$ sub-sequences of length $\mathrm{n}$, and the th sample element of the mth sub-sequence is recorded as $\mathrm{x}_{\mathrm{i}, \mathrm{m}}(\mathrm{t}=1,2, \cdots, \mathrm{n} ; \mathrm{m}=1,2, \cdots, \mathrm{M})$, then the rescaled range statistic of the mth sub-sequence is as follows:

$$
(R / S)_{n, m}=\frac{R_{n, m}}{S_{n, m}}
$$
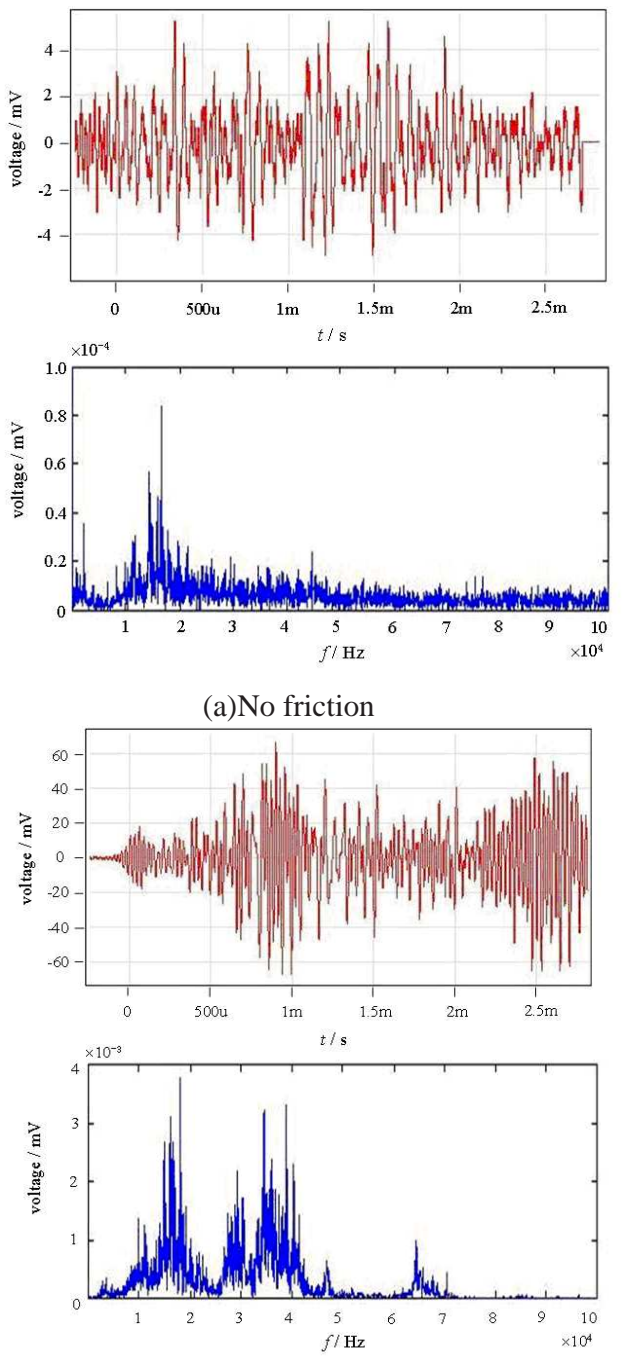

(b)Slight friction
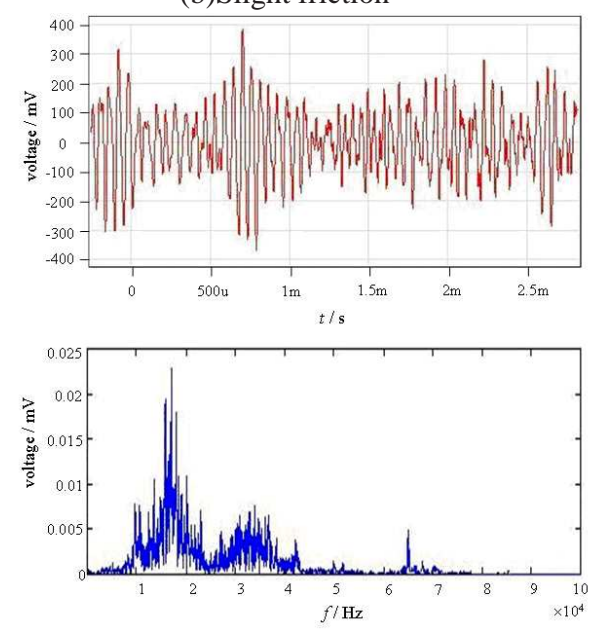

(c)Strong friction

Fig. 4: AE waveforms and spectra of different friction states 
In the formula, $R_{n, m}$ stands for the range of the mth sub-sequence; $S_{n, m}$ stands for the standard deviation of the mth sub-sequence:

$$
\begin{gathered}
R_{n, m}=\max _{1 \leq k \leq n} \sum_{t=1}^{k}\left(x_{t, m}-\overline{x_{n, m}}\right)-\min _{1 \leq k \leq n} \sum_{t=1}^{k}\left(x_{t, m}-\overline{x_{n, m}}\right) \\
S_{n, m}=\sqrt{\frac{1}{n} \sum_{t=1}^{n}\left(x_{t, m}-\overline{x_{n, m}}\right)^{2}}
\end{gathered}
$$

The $\overline{x_{n, m}}$ in the formula stands for the mean value of the mth sub-sequence:

$$
\overline{x_{n, m}}=\frac{1}{n} \sum_{t=1}^{n} x_{t, m}
$$

Then the rescaled range of the time series $\left\{x_{t}\right\}_{t=1}^{\mathrm{T}}$ corresponding to the scale length $\mathrm{n}$ is as follows:

$$
(R / S)_{n}=\frac{1}{M} \sum_{m=1}^{M}(R / S)_{n, m}
$$

We can get a series of corresponding rescaled ranges by choosing several $\mathrm{n}$ with different length. There is a relationship between the rescaled range sequence $(R / S)_{n}$ and its corresponding scale length $\mathrm{n}$ as follows:

$$
(\mathrm{R} / \mathrm{S})_{\mathrm{n}}=\mathrm{C}_{\mathrm{n}}^{\mathrm{H}}
$$

In the formula: $\mathrm{C}$ is a constant; $\mathrm{H}$ stands for the Hurst index. Through taking double logarithm:

$$
\lg (\mathrm{R} / \mathrm{S})=\lg \mathrm{C}+\mathrm{Hlgn}
$$

Adopt the least square fitting to formula (23), and we can reckon up the Hurst index.

If $0 \leq \mathrm{H}<0.5$, the time series signals show an anti-continuing act i.e. if the system appears a growth trend in a given period then it may shows a declining trend in the next period. If $0.5<\mathrm{H}<1$, the time series signals show a continued stability i.e. the present change has a lasting impact on the following changes. If the system shows a growth trend in a period, then it will continue to maintain this trend. If $\mathrm{H}=0.5$, the time series signal is a signal generated by an independent free process i.e. the present change has no impact on the following changes and the time series is random. If $H=1$, the future condition can be completely predicted by the current situation and the time series is a straight line.

It is can be seen from Figure 5 that the Hurst index under the condition of no friction and slight friction is less than 0.5 which indicates that the friction acoustic emission signals have an anti-sustained behavior. This is because most signal consists of the background mechanical noise generated by the motor vibration under

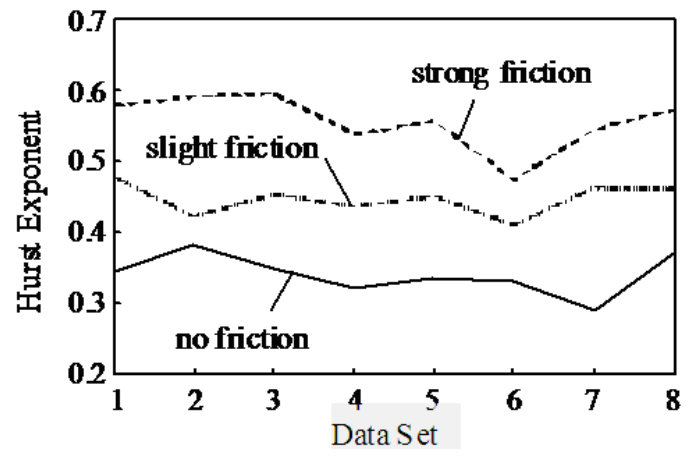

Fig. 5: The Hurst indexes under different friction states

no friction condition, and the signal appears an inversion recovery phenomenon showing anti-persistence as is shown in Figure 3(a); under light friction condition, the stability of periodic friction is not sufficient enough to cover up the anti-persistent of background mechanical noise, so that the entirety shows an anti-persistence, but at this moment, the signal has an intrinsic potential of tending to be persistent, thus the Hurst index of the signal at this moment is bigger than that in the no friction state; in a strong friction state, the regular friction makes the overall signal show a kind of stable trend, and the Hurst index is greater than 0.5 which illustrates such a stable trend. We can see that the Hurst index can commendably distinguish the different state of friction.

\subsection{Approximate entropy (ApEn)}

Approximate entropy measures the probability of the new model generated in the signal mainly from the point of view of measuring the complexity of the time series and it has also been widely used in the field of fault diagnosis in recent years. Approximate entropy has a better noise immunity whose size is proportional to the complexity of the sequence, and it is a suitable characteristic parameter to characterize the intensity and complexity of AE signal whom with a nonlinear characteristic and is susceptible to noise interference, and it measures the complexity of time series only from the statistical aspect, the advantages that it only needs a shorter data and a shorter period of time to obtain the computing result makes it easier to meet the requirements of real-time analysis of AE signals [16] .

Assume that the collected original data is $\{\mathrm{u}(\mathrm{i}), \mathrm{i}=0,1, \cdots, \mathrm{N}\}$, the approximate entropy can be calculated by the following steps with the given model dimension $\mathrm{m}$ and similar tolerance $\mathrm{r}$.

1) Constitute the m-dimensional vector $X(i)$ orderly with the sequence $\{u(i)\}$ i.e.:

$\mathrm{X}(\mathrm{i})=[\mathrm{u}(\mathrm{i}), \mathrm{u}(\mathrm{i}+1), \cdots, \mathrm{u}(\mathrm{i}+\mathrm{m}-1)], \mathrm{i}=1, \cdots, \mathrm{N}-\mathrm{m}+1$ 
2) Calculate the distance between vector $X(i)$ and its coversed sine $\mathrm{X}(\mathrm{j})$ for each value of $\mathrm{i}$ :

$$
\mathrm{d}[\mathbf{X}(i), \mathbf{X}(j)]=\max _{k=0}^{m-1}|u(i+k)-u(j+k)|
$$

3) Calculate the number of $d[X(i), X(j)]<r$ and the ratio of it and the total number vector $\mathrm{N}-\mathrm{m}+1$, and the result can be recorded as $\mathrm{C}_{\mathrm{i}}^{\mathrm{m}}(\mathrm{r})$ i.e.:

$$
\mathrm{C}_{\mathrm{i}}^{\mathrm{m}}(\mathrm{r})=\{\operatorname{TheSumofd}[\mathrm{X}(\mathrm{i}), \mathrm{X}(\mathrm{j})]<\mathrm{r}\} /(\mathrm{N}-\mathrm{m}+1)
$$

4) First take the logarithm of $C_{i}^{m}(r)$, and then calculate its average value for all $\mathrm{i}$, and the result can be recorded as $\Phi^{\mathrm{m}}(\mathrm{r})$ i.e.:

$$
\Phi^{m}(r)=\frac{1}{N-m+1} \sum_{i=1}^{N-m+1} \ln C_{i}^{m}(r)
$$

5) Then repeat the process in formula (24) to (27) on $\mathrm{m}+1$, and we can get $\Phi^{\mathrm{m}+1}(\mathrm{r})$.

6) In theory, the approximate entropy of this sequence is as follows:

$$
A_{p E n}(m, r)=\lim _{N \rightarrow \infty}\left[\Phi^{m}(r)-\Phi^{m+1}(r)\right]
$$

$\mathrm{N}$ can not be $\infty$ in actual projects, when $\mathrm{N}$ is a finite value, the result obtained by the above steps is the estimated value of approximate entropy (ApEn) while the sequence length is $\mathrm{N}$. and the result can be recorded as follows:

$$
\mathrm{A}_{\mathrm{pEn}}(\mathrm{m}, \mathrm{r}, \mathrm{N})=\Phi^{\mathrm{m}}(\mathrm{r})-\Phi^{\mathrm{m}+1}(\mathrm{r})
$$

The value of $A_{p E n}$ is clearly related to that of $\mathrm{m}$ and $\mathrm{r}$ which are usually made that $\mathrm{m}=2$ and $\mathrm{r}=0.1 \approx 0.25 \mathrm{~S}_{\mathrm{D}}$ $\left(S_{D}\right.$ is the standard deviation of sequence $\{\mathrm{u}(\mathrm{i})\}$ ).

The application of the approximate entropy in the analysis of the friction $\mathrm{AE}$ signal has the following advantages:

1) Approximate entropy has a better noise immunity, especially the better ability to withstand the randomly generated transient strong interference. Its noise immunity is very helpful for the characteristic extraction of the AE signal which is susceptible to strong noise interference.

2) The approximate entropy is proportional to the complexity of the sequence, the more complex the sequence is, the greater the corresponding approximate entropy becomes. When it goes to the friction AE signals, the greater the friction strength is, the worse the regularity of the signal appears, the higher the complexity shows, and the greater the corresponding approximate entropy becomes.

3) Approximate entropy algorithm consists of a greater amount of information than the mean, variance and other general statistical parameters do because it just contains the information of the time signal sequence in the distribution pattern while the mean, variance and other statistical parameters missed them, thus it can more effectively extract the characteristics in the friction signals.

4) Approximate entropy measures the complexity of time series only from the statistical point of view, thus it only need a shorter data and a short period of time to obtain the right result and it easy to meet the real-time requirements of AE signals. Figure6 shows the different approximate entropy curves under different friction strength shown in Figure 3.

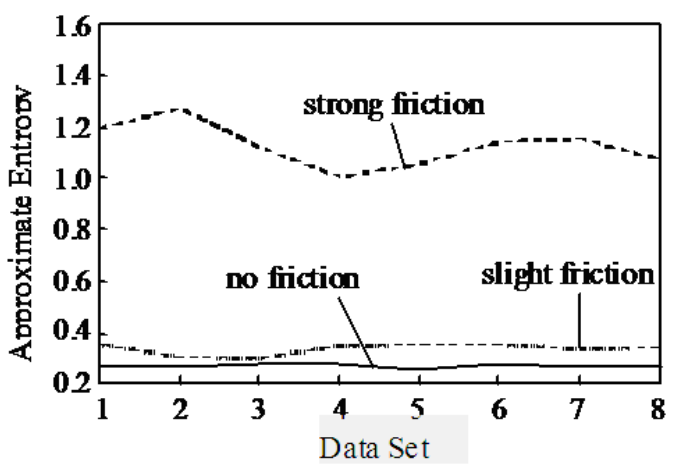

Fig. 6: Approximate entropy of different friction states

We can see from Figure 6 that the approximate entropy gradually becomes larger with the improvement of the frictional strength, and its distribution of each friction state is homogeneous. Under no friction state, the signal is the background mechanical noise with low complexity and the minimum corresponding approximate entropy; under slight friction state, even though the friction strength is small, the signal complexity of incentive AE signals is bigger than that in no friction state, the approximate entropy increases and it can be distinguished from no friction state; In the state of strong friction, the friction source produces large amounts of high-strength friction AE signals with substantially increasing signal complexity and the largest corresponding approximate entropy which is far outweigh that of the slight friction state.

\section{Experimental analysis}

According to the above analysis, the average amplitude, the maximum amplitude, the amplitude dynamic range, the Hurst exponent and approximate entropy (ApEn) of the AE signals are selected as its identification characteristic parameters in this paper. In order to avoid the influence of noise, we only consider the average of the amplitude absolute value of the signal amplitude which 
exceeds a certain threshold when we analyze the characteristics such as the average amplitude and dynamic range etc.

During the test, separately select three groups of AE signals with the time length $3000 \mathrm{~ms}$ from each of the three states of no friction, slight friction and strong friction state when the rotor speed is respectively 500 rpm, $1200 \mathrm{rpm}$ and $2150 \mathrm{rpm}$, and there are totally 27 sets of data, extract $\mathrm{AE}$ characteristic parameters from each set of data to form a parameter vector $U=\left\{x_{1}^{i}, x_{2}^{i}, \ldots, x_{J}^{i}\right\}$, and identify the friction state by making use of the Gaussian mixture model based on embedded self-associative neural network. In order to compare the effectiveness of the proposed identification method in this paper, here we have compared it with the friction state identification result of the baseline-based Gaussian mixture background model. Make the no friction equals 0 , slight friction equals 1 and strong friction equals 2 , then the identification result is as shown in Table 1.

Table 1: The friction state identification results of the two models

\begin{tabular}{|c|c|c|c|c|}
\hline Data set & $\begin{array}{c}\text { Rotational } \\
\operatorname{speed}(\mathrm{r} / \mathrm{min})\end{array}$ & $\begin{array}{c}\text { The actual } \\
\text { state }\end{array}$ & GMM & $\begin{array}{c}\text { GMM } \\
+ \text { TDNN }\end{array}$ \\
\hline 1 & 500 & 0 & 0 & 0 \\
\hline 2 & 500 & 0 & 0 & 0 \\
\hline 3 & 500 & 0 & 0 & 0 \\
\hline 4 & 500 & 1 & 1 & 1 \\
\hline 5 & 500 & 1 & 1 & 1 \\
\hline 6 & 500 & 1 & 0 & 1 \\
\hline 7 & 500 & 2 & 2 & 2 \\
\hline 8 & 500 & 2 & 2 & 2 \\
\hline 9 & 500 & 2 & 2 & 2 \\
\hline 10 & 1200 & 0 & 0 & 0 \\
\hline 11 & 1200 & 0 & 1 & 0 \\
\hline 12 & 1200 & 0 & 0 & 0 \\
\hline 13 & 1200 & 1 & 1 & 1 \\
\hline 14 & 1200 & 1 & 1 & 1 \\
\hline 15 & 1200 & 1 & 1 & 1 \\
\hline 16 & 1200 & 2 & 2 & 2 \\
\hline 17 & 1200 & 2 & 2 & 2 \\
\hline 18 & 1200 & 2 & 2 & 2 \\
\hline 19 & 2150 & 0 & 0 & 0 \\
\hline 20 & 2150 & 0 & 1 & 1 \\
\hline 21 & 2150 & 0 & 0 & 0 \\
\hline 22 & 2150 & 1 & 2 & 2 \\
\hline 23 & 2150 & 1 & 1 & 1 \\
\hline 24 & 2150 & 1 & 1 & 1 \\
\hline 25 & 2150 & 2 & 2 & 2 \\
\hline 26 & 2150 & 2 & 2 & 2 \\
\hline 27 & 2150 & 2 & 2 & 2 \\
\hline \multicolumn{3}{|c|}{ Friction existence identification rate } & $96 \%$ & $100 \%$ \\
\hline \multicolumn{3}{|c|}{ Friction state recognition rate } & $85 \%$ & $93 \%$ \\
\hline
\end{tabular}

As can be seen from Table 1, both models have very good identification results when determine whether there is friction or not, and during the identification of the specific state of friction, the identification method of the time delay embedded Gaussian mixture model network has achieved a certain improvement on the identification efficiency over the Gaussian mixture model identification method, and it indicates that it can improve the identification efficiency of the AE signal by adding the time delay network. The main reason of the decreased efficiency of friction state identification is that with the increasing rotating speed, the noise generated by the test bed itself is enhanced, and the energy level in slight friction state is also increased, as a result, the identification deviation of time-domain characteristic parameters occurs.

\section{Conclusion}

This paper makes it possible to learn time information of feature vectors, increase the acoustic emission signal likelihood probability of target rotor friction and balance the requirements of the maximum likelihood probability criteria by embedding a TDNN network in GMM which is equivalent to transforming the feature domain and model domain of GMM simultaneously and also plays a better role in eliminating the noise effect in noisy environments. We have solved the model learning method by using a two-stage iterative method. When the weight coefficient of neural network output layer is 0 , the model degenerates into GMM. We have chosen the average amplitude, maximum amplitude, amplitude dynamic range, the Hurst index and approximate entropy of the friction acoustic emission signals to constitute the parameter vector after experimental and theoretical analysis. The experimental results show that this method has a high friction fault recognition rate, and its recognition effect is higher than that of the conventional GMM.

\section{Acknowledgement}

This work was supported by the Natural Science Foundation of China under Grant No.51075068, No. 60872073, No.60975017.

\section{References}

[1] Green A, Characteristics of Acoustic Emission Response from MaterialsJapan Acoustic Emission Symposium, Tokyo, 232-235 (1992).

[2] Terchi L, Time-frequency analysis of acoustic emissionEngineering Fracture Mechanics, 69717-728 (2002).

[3] Deng AidongGao WeiYang Jianganget al, Application of discrete fractional cosine transform in acoustic emission signal de-noiseProceedings of the CSEE, 28, 72-76 (2008). 
[4] Adams M LAbu Mahfouz , Expletory Research Chaos Concepts as Diagnostic Tools for Assessing Rotating Machinery Vibration SignaturesProceedings of the Fourth International Conference on Rotor Dynamics, Chicago, USA, 29-39 (1994).

[5] D. A. Reynolds and R. C. Rose, Robust Text-independent Speaker Identification Using Gaussian Mixture Speaker Models. IEEE Trans. Speech Audio Processing, 3, 72-83 (1995).

[6] D. Reynolds, T. Quatieri and R. Dunn, Speaker Verification Using Adapted Gaussian Mixture Models [J]. Digital Signal Processing, 10, 19-41 (2000).

[7] Soonil Kwon and Shrikanth Narayanan, Robust Speaker identification based on selective use of featue vectors [J]. Pattern Recognition Letters, 28, 85-89 (2007).

[8] W. M. Campbell, D. E. Sturim, D. A. Reynolds, SVM based speaker verification using A GMM supervector kernel and NAP variability compensation [C]. Proceedings of ICASSP. IEEE, 1, 97-100 (2006).

[9] Shou-Chun Yin, Richard Rose, and Patrick Kenny, A joint factor analysis approach to progressive model adaptation in Text-Independent speaker verification [J]. IEEE Trans. on Audio, Speech and Language Processing, 15, 1999-2110 (2007).

[10] M. W. Mak, W. G. Allen and G. G. Sexton. Speaker identification using multilayer perceptron and radial basis function networks. Neurocomputing, 6, 99-117 (1994).

[11] Sachin Joshi, Kishore Prahallad, B. Yegnanarayana, TDNN-HMM Models for Speaker Verification and Speech Recognition, in Proceedings of International Joint Conference on Neural Networks, (2008).

[12] Bennani Y and Gallinari P, on the use of TDNN-Extracted Features Information In Talker Identification. Proceedings of ICASSP, 385-388 (1991).

[13] Dev and Amita, Effect of retroflex sounds on the recognition of Hindi voiced and unvoiced stops. AI \& SOCIETY, 23, 603-612 (2009).

[14] Huiyu Zhou, Taj, M. and Cavallaro, A, Target Detection and Tracking With Heterogeneous Sensors, IEEE Journal of Selected Topics in Signal Processing, 2, 503-513 (2008).

[15] D. ERumelhart, G. E. Hinton and R. J. Williams, Learning representations by back-propagating errors, Nature, 323, 533-536 (1986).

[16] JIANG ZhangDENG Aidong Identification of rotating machinery rub and impact fault using Hurst exponent and approximate entropy as characteristic parameters of acoustic emission [J]Proceedings of the CSEE, 30, 96-102 (2010) (in Chinese).

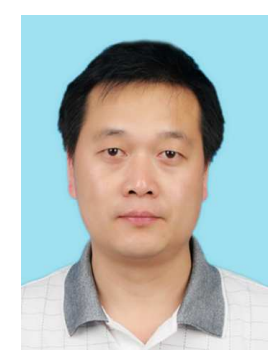

Aidong Deng was born in 1968. He received his B.E. degree in Industrial Electric Automation from Harbin Shipbuilding Engineering Institute in 1991, the M.S. degree in 1994, and the Ph.D. degree in 2008, both from Southeast University. Currently, he is an associate professor with the National Engineering Research Center of Turbo-generator Vibration of School of Energy \& Environment, Southeast University, Nanjing, China. He worked in areas of measurement technology and instruments, fault analyses and signal processing. Recently he has been working on acoustic emission technology for rotating machinery fault diagnosis applications.

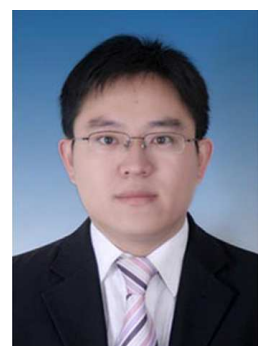

Hao Cao was born in 1982. He received his doctorate from the Department of Power Engineering, Southeast University, in 2012 and is currently working at the Hunan Electric Power Corporation Research Institute in Changsha, China. His research areas are rotating machinery fault monitoring and diagnosis, and rotor dynamics and flow-induced vibration.

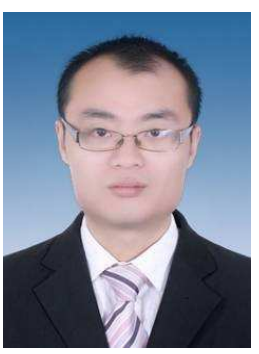

Hang Tong was born in 1987. He received his B.E. degree from China University of Mining and Technology in 2009 and the M.S. degree from Southeast University in 2012. His research interests include power engineering and fault analyses. 


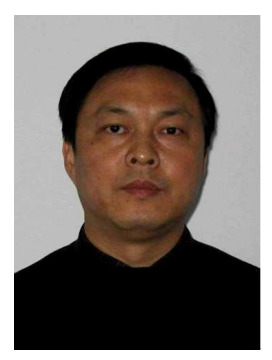

Li Zhao was born in 1958 . He received his B.E. degree from Nanjing University of Aeronautics and Astronautics in 1982, the M.S. degree from Suzhou University in 1988, and the Ph.D. degree from Kyoto Institute of Technology, Japan, in 1998. Currently, he is a Professor with the School of Information Science and Engineering at Southeast University. His major interests include speech signal processing, emotional information processing, pattern recognition and natural language processing.

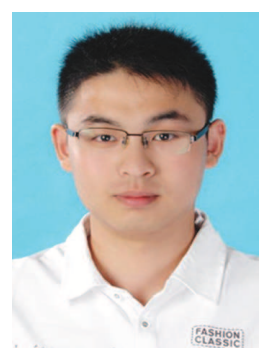

\begin{abstract}
Kang Qin was born in 1988. He received his B.E. degree from Nanjing University of Technology in 2011. Now he is a master degree candidate in Southeast University. His research interests include fault analyses and acoustic emission technology.
\end{abstract}

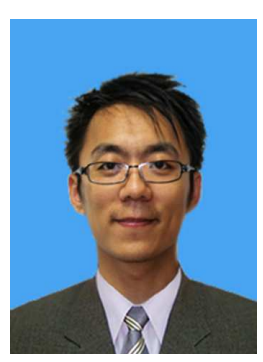

Xin Yan was born in 1989. He received his B.E. degree from Suzhou University in 2011. Now he is a master degree candidate in Southeast University. His research interests include fault analyses and acoustic emission technology. 